ARTICLE

\title{
Fluence-to-Dose Conversion Coefficients for Muons and Pions Calculated Based on ICRP Publication 103 Using the PHITS Code
}

\author{
Tatsuhiko SATO ${ }^{1, *}$, Akira ENDO ${ }^{1}$ and Koji NIITA ${ }^{2}$ \\ ${ }^{1}$ Japan Atomic Energy Agency, 2-4 Shirakata-Shirane, Tokai-mura, Naka-gun, Ibaraki-ken, 319-1195, Japan \\ ${ }^{2}$ Research Organization for Information Science and Technology, \\ 2-4 Shirakata-Shirane, Tokai-mura, Naka-gun, Ibaraki-ken,319-1106, Japan
}

\begin{abstract}
The fluence to effective-dose and organ-absorbed-dose conversion coefficients for charged pions and muons were calculated based on the instructions given in ICRP Publication 103. For the calculation, the particle motions in the ICRP/ICRU adult reference computational phantoms were simulated using the PHITS code for four idealized irradiation geometries as well as those closely representing the geometrical situations of cosmic-ray muon exposure. Cosmic-ray pion and muon dose rates over a wide altitude range were estimated using the calculated dose conversion coefficients. The results of the calculation indicate that the assumption of the isotropic irradiation geometry is suitable to be utilized in the dose estimations for cosmic-ray pions and muons. It is also found from the calculation that the introduction of ICRP103 gives little impact on the pion and muon dosimetries, since the radiation weighting factors assigned to those particles are maintained in the issue.
\end{abstract}

KEYWORDS: aircrew dosimetry, dose conversion coefficients, PHITS, muon, pion, cosmic-ray, voxel phantom

\section{Introduction}

Recently, the International Commission on Radiological Protection (ICRP) released a new set of fundamental recommendations for a system of radiological protection in ICRP Publication $103 .{ }^{1)}$ In the new recommendations, the radiation and tissue weighting factors, referred to as $w_{\mathrm{R}}$ and $w_{\mathrm{T}}$ respectively, were updated based on the latest scientific information on the biology and physics of radiation exposure. In addition, ICRP decided to introduce sex-specific voxel phantoms to represent the Reference Male and Reference Female ${ }^{2)}$ (hereafter referred to as ICRP/ICRU reference phantoms) for the forthcoming update of the organ dose coefficients for both internal and external radiation sources. Such phantoms are the result of recent efforts by the radiation protection community to adopt anatomically realistic medical images and powerful computer technologies. ${ }^{3)}$

Following the instruction given in ICRP103, a set of the fluence to the effective-dose conversion coefficients for neutrons, ${ }^{4)}$ protons ${ }^{4)}$ and heavy ions ${ }^{5}$ over a wide energy range had been calculated, using the PHITS code. ${ }^{6,7)}$ Those data are indispensable for estimating the doses for astronauts in space as well as workers in nuclear and accelerator facilities, and their numerical values were partially released to the public via the internet as the electric database DDCC. ${ }^{8)}$ On the other hand, the evaluation of the conversion coefficients for charged pions and muons are necessary for aircrew dosimetry, since cosmic-rays can generate a number of charged pions by causing nuclear interactions in the atmosphere, and most of them decay into muons and neutrinos. Their dose conversion coefficients were however calculated ${ }^{9,10)}$ based

*Corresponding author, E-mail: sato.tatsuhiko@jaea.go.jp

(C) 2011 Atomic Energy Society of Japan, All Rights Reserved. only on ICRP Publication $60^{11)}$ and no dataset was available for the values based on ICRP103.

We therefore calculated the conversion coefficients from fluence to the effective dose and organ absorbed doses for charged pions and muons on the basis of ICRP103. The particle motions in the ICRP/ICRU reference phantoms were simulated by PHITS for four idealized irradiation geometries, anterior-to-posterior (AP), posterior-to-anterior (PA), isotropic (ISO), and semi-isotropic from the upper hemisphere (SS-ISO). For muons, the PHITS simulations were also carried out for the irradiation geometries closely representing the geometrical situations of cosmic-ray muon exposure, which are referred to as Aircrew-Exposure (AE) geometries in this paper. For charged pions, the human-body-averaged mean quality factors were calculated using the $Q(L)$ relationship defined in ICRP60, in order to examine the adequacy of their $w_{\mathrm{R}}$ value. The impact of the introduction of ICRP103 on aircrew dosimetry was analyzed by comparing between their ICRP60- and ICRP103-based effective dose rates due to charged pion and muon exposure.

\section{Calculation Procedure}

A detailed procedure for calculating the dose conversion coefficient as well as the mean quality factor has been reported in our previous paper. ${ }^{4)}$ Thus, only a brief explanation of the procedure is given in this article.

The PHITS simulations were performed to analyze the particle motions in the ICRP/ICRU reference phantoms irradiated by mono-energetic charged pions and muons. The incident energies were changed from $1 \mathrm{MeV}$ up to $200 \mathrm{GeV}$. The four idealized geometries (AP, PA, ISO and SS-ISO), 


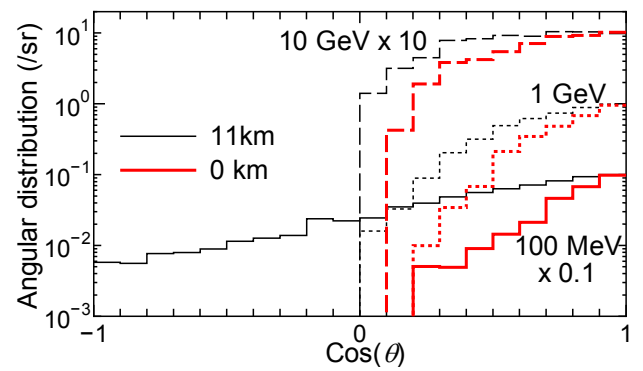

Fig. 1 Angular distributions of cosmic-ray muons at a flight altitude $(11 \mathrm{~km})$ and sea level $(0 \mathrm{~km})$ calculated by PHITS. These data are normalized to 1 at the vertical direction.

together with the two AE geometries were assumed in the simulations, although those for the realistic conditions were carried out only for muon exposure. For the AE geometries, the angle of incident muons was sampled following the angular distributions of cosmic-ray muons at the altitudes of 0 and $11 \mathrm{~km}$ at the polar region for the solar minimum, which were calculated by performing an atmospheric propagation simulation of cosmic-rays using PHITS. ${ }^{12)}$ A more detailed description of this source-determination procedure is given elsewhere. ${ }^{13)}$ As examples, the angular distributions of negative muons for energies around $100 \mathrm{MeV}, 1 \mathrm{GeV}$ and $10 \mathrm{GeV}$ are depicted in Fig. 1. It can be seen from the graph that the vertical directivities are more apparently observed in the data at sea level in comparison to those at the flight altitude.

For high-energy irradiation, it should be noted that the delta-ray equilibrium is not established near the irradiation surface of the human body placed in vacuum. Thus, the deposition energies slightly decrease at shallower locations in the human body due to the production of delta-rays. The equilibrium is, however, established in the realistic exposure conditions, since the human body is surrounded by air. Therefore, the delta-ray production was ignored in the PHITS simulation in order to estimate the deposition energy in the human body under situations closer to reality.

The absorbed doses per unit fluence in each organ or tissue, $D_{\mathrm{T}}$, and their probability densities in terms of LET, $d_{\mathrm{T}}(L)$, were derived from the PHITS simulation. The conversion coefficients from fluence to the effective dose, $E$, can be calculated from $D_{\mathrm{T}}$ with $w_{\mathrm{R}}$ and $w_{\mathrm{T}}$ updated in ICRP103, using the equation:

$$
E=\sum_{\mathrm{T}} w_{\mathrm{T}} H_{\mathrm{T}}=w_{\mathrm{R}} \sum_{\mathrm{T}} w_{\mathrm{T}} D_{\mathrm{T}},
$$

where $H_{\mathrm{T}}$ denotes the equivalent dose in organ or tissue per unit fluence, which can be simply determined from $D_{\mathrm{T}}$ multiplied with $w_{R}$. Note that the numerical values of $w_{R}$ assigned to charged pions and muons are 2 and 1, respectively.

The human-body-averaged mean quality factor, $q_{\mathrm{E}}$, can be obtained by the equation:

$$
q_{\mathrm{E}}=\sum_{\mathrm{T}} w_{\mathrm{T}} H_{\mathrm{T}, L} / \sum_{\mathrm{T}} w_{\mathrm{T}} D_{\mathrm{T}},
$$

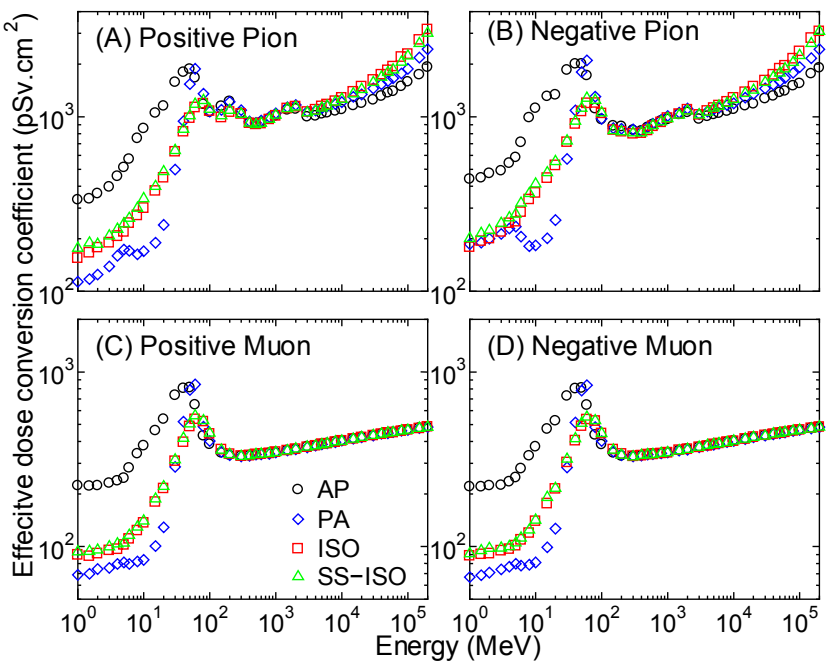

Fig. 2 Conversion coefficients from fluence to the effective dose calculated based on ICRP103 for charged pions and muons for the AP, PA, ISO and SS-ISO irradiation geometries.

where $H_{\mathrm{T}, L}$ denotes the $Q(L)$-based organ dose equivalent per unit fluence, which can be determined by

$$
H_{\mathrm{T}, L}=D_{\mathrm{T}} \int_{L} Q(L) d_{\mathrm{T}}(L) \mathrm{d} L .
$$

\section{Results and Discussion}

\section{Fluence to Effective Dose Conversion Coefficients}

Figure 2 shows the calculated conversion coefficients from fluence to the effective dose for charged pions and muons for the four idealized irradiation geometries. The statistical uncertainties of these data are generally small - less than $5 \%$ in most cases.

As can be seen from the graphs, the energy dependences of the conversion coefficients have a peak structure around $50-100 \mathrm{MeV}$. Below the peak energy, a majority of incident particles stop inside the human body, and deposit most of their energies within a certain distance from the stopped point. Thus, the conversion coefficients significantly depend on the irradiation geometry at lower energies. At the peak energy, the range of incident pions and muons coincide with the average depths from the irradiated surface to the organs with high $w_{\mathrm{T}}$ value such as the stomach and red bone marrow.

Above the peak energy, on the other hand, the incident particles have such long ranges that they generally penetrate the human body. Hence, the doses are more uniformly distributed inside the human body in comparison to the low-energy irradiations. The irradiation geometry dependence, however, can still be observed in the conversion coefficients for high-energy pions, where the AP and ISO irradiations give the minimum and maximum values, respectively. This tendency is due to the fact that such high-energy pions can trigger a cascade of secondary particles by successively inducing complex nuclear reactions, and deposit more energy to locations deeper from the irradiation surface. 


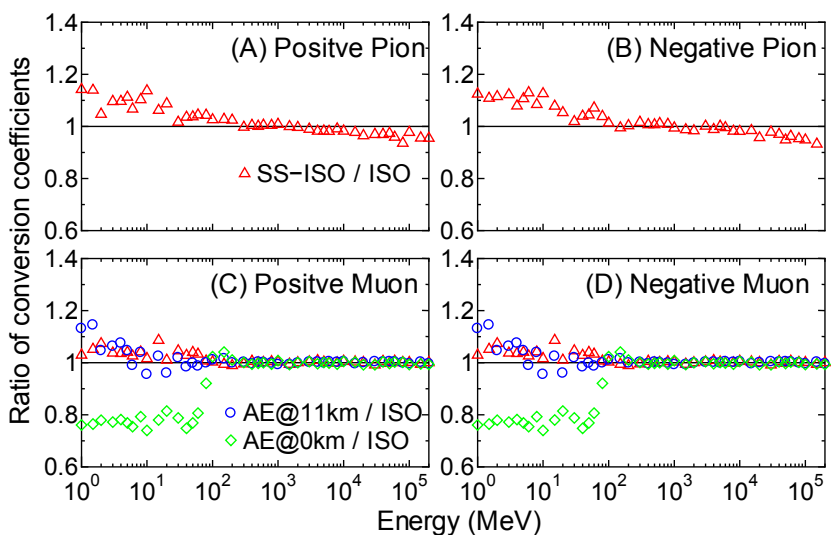

Fig. 3 Ratios of the effective-dose conversion coefficients for the SS-ISO and AE geometries to the corresponding data for the ISO geometry. AE@0km and AE@11km denote the aircrew-exposure geometries based on the angular distributions of the cosmic-ray muon fluxes at the altitudes of 0 and $11 \mathrm{~km}$, respectively.

In order to discuss the adequacy of the ISO assumption utilized in the aircrew dosimetry, the ratios of the conversion coefficients for the SS-ISO geometry to the corresponding data for the ISO geometry are plotted in Fig. 3. Note that SS-ISO is generally considered as a more realistic geometry in comparison to ISO for representing the geometrical situation of cosmic-ray exposure in the atmosphere. For muon irradiations, the ratios of the data for the $\mathrm{AE}$ geometries are also shown on the graph. Panels (A) and (B) show the ratios of the conversion coefficients for pions decrease with the increase of the incident energy. This tendency is attributed to the facts that radiologically sensitive organs are generally positioned at the upper side of the human body, and lowerand higher-energy pions deposit more energy to the organs located close to and far away from the irradiation surface, respectively.

For muons, similar tendencies are observed in the data for the SS-ISO as well as the AE-at- $11 \mathrm{~km}$ geometries for lower energies. On the other hand, the ratios of the data for the AE-at-0km geometry are smaller than 1 for the energies below $80 \mathrm{MeV}$. This is because the angular distribution of cosmic-ray muons at sea level is considerably vertical even at low energies so that most of them are incident from the head and not able to reach to the torso, where many radiologically sensitive organs are positioned. For higher energies, the ratios become very close to 1 for all the irradiation geometries, since the doses are uniformly distributed inside the human body, as discussed before.

\section{Human-Body-Averaged Mean Quality Factors}

Figure 4 shows the human-body-averaged mean quality factors for pions calculated by Eq. (2). The irradiation geometry dependences of the mean quality factors are not so significant, although only the data for the ISO geometry are depicted in the graph. The $w_{\mathrm{R}}$ value assigned to pions is also shown in the graph for a comparison.

It is evident from the graph that the mean quality factors

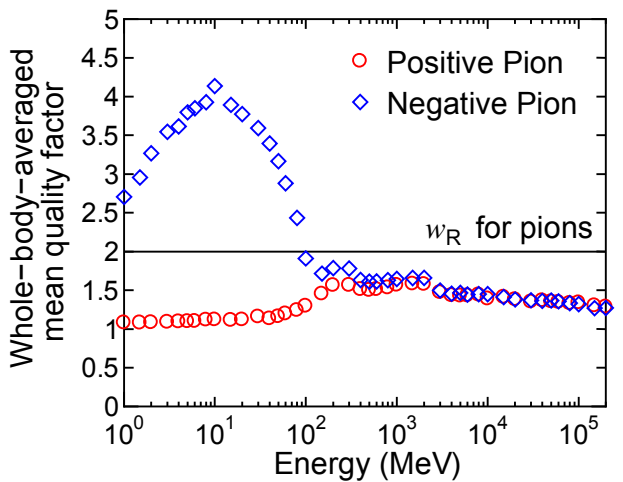

Fig. 4 Human-body-averaged mean quality factors for pions of the ISO geometry.

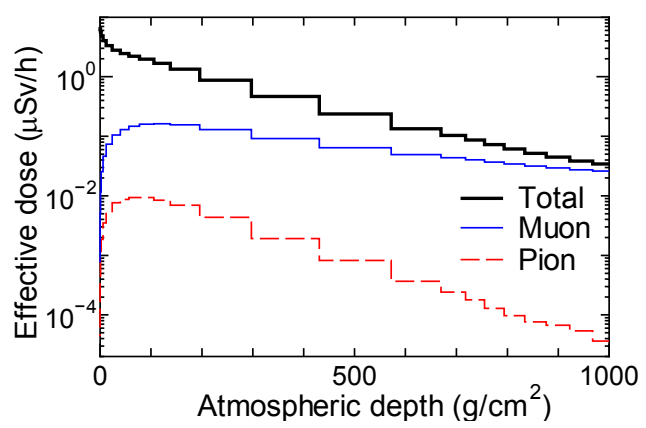

Fig. 5 Atmospheric-depth dependences of the cosmic-ray pion, muon and total dose rates calculated using the effective-dose conversion coefficients for the ISO geometry.

for positive and negative pions generally agree with each other for high energies, and they are smaller than the $w_{R}$ value. For low energies, on the other hand, the data for negative pions are much larger than the respective data for positive pions. The disagreement at the low-energy region is triggered by difference in the treatment of stopped pions in the PHITS code, where negative pions are forced to interact with surrounding nucleus, while positive pions are to decay into a muon and neutrino. Thus, most of the deposition energies are imparted by muons and their descendants for the low-energy positive-pion irradiations, and consequently, their mean quality factors become very close to 1 . On the other hand, secondary charged particles with high LET play the dominant role in the energy deposition process for the low-energy negative-pion irradiations. Hence, their mean quality factors become much higher than those for positive pions as well as the $w_{R}$ value for energies below $100 \mathrm{MeV}$. This numerical incompatibility, however, does not cause a significant influence in aircrew dosimetry, since the low-energy pion fluxes are generally much lower than the corresponding high-energy fluxes at all altitudes.

\section{Calculation of Aircrew Doses}

Figure 5 shows the atmospheric-depth dependences of the cosmic-ray pion and muon dose rates calculated using the effective-dose conversion coefficients for the ISO geo- 


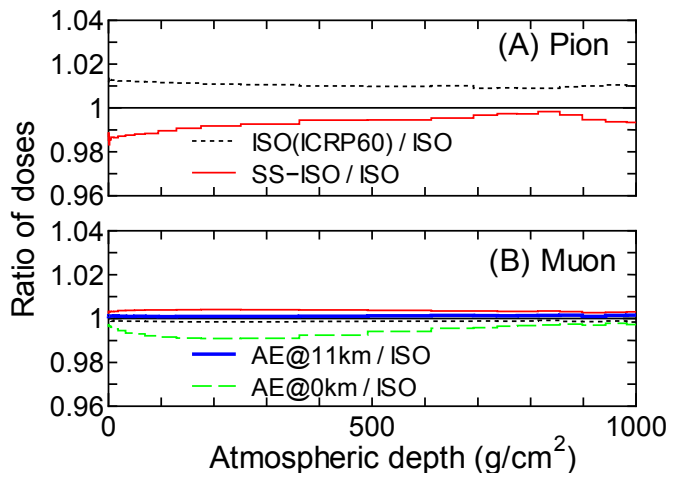

Fig. 6 Ratios of the cosmic-ray pion and muon dose rates calculated using the effective-dose conversion coefficients for the SS-ISO (red solid line) and AE geometries (thick blue and green dashed lines) to the corresponding data for the ISO geometry, as a function of the atmospheric depth. The ratios of the effective dose rates based on ICRP60 to those based on ICRP103 are also drawn in the graph as black dotted line.

metry. The total dose rates are also shown in the graph. In the calculation, the cosmic-ray fluxes were estimated from the PHITS simulation of the atmospheric propagation of cosmic-rays. ${ }^{12)}$ It is evident from the graph that the muon contributions are dominant over the total dose rates near sea level, and pion contributions are less than $1 \%$ of the total at all altitudes. These tendencies are the same as those obtained from a previous study of ours ${ }^{12)}$ using the conversion coefficients for the effective dose based on ICRP60.

Figure 6 depicts the ratios of the cosmic-ray pion and muon dose rates calculated using the effective-dose conversion coefficients for the SS-ISO and AE geometries to the corresponding data for the ISO geometry, as a function of the atmospheric depth. The ratios of the effective dose rates based on ICRP60 to those based on ICRP103 are also shown in the graph. The conversion coefficients for the ICRP60-based effective dose adopted in this calculation were derived from the organ-absorbed-dose conversion coefficients obtained from this study coupled to the $w_{\mathrm{R}}$ and $w_{\mathrm{T}}$ values defined in ICRP60. Note that the $w_{\mathrm{R}}$ values for pions and muons given in ICRP60 are the same as those in ICRP103, and thus, the difference of the two conversion coefficients are attributed only to the difference of the $w_{\mathrm{T}}$ values.

The graphs show that the ratios are very close to 1 for all the plotted geometries, indicating the suitability of the ISO geometry to be employed in the cosmic-ray pion and muon dosimetry. This is because the majority of cosmic-ray pion and muon doses consist of contributions from high-energy particles whose dose conversion coefficients are almost independent of the irradiation geometry, as shown in Fig. 4. The ICRP60-based effective dose rates are larger than the ICRP103-based data by approximately 1\%, since ICRP increased the numerical values of $w_{\mathrm{T}}$ assigned to the breast from 0.05 to 0.12 in ICRP103, where its organ-absorbed-dose conversion coefficients are smaller than those for other organs for high-energy pion irradiations. The effect of this difference is, however, almost negligible in the aircrew dose estimation, since the pion contributions are less than $1 \%$ of the total at all altitudes, as shown in Fig. 5.

\section{Conclusions}

The fluence to effective-dose and organ-absorbed-dose conversion coefficients for charged pions and muons were calculated using the PHITS code and ICRP/ICRU reference phantoms, following the instruction given in ICRP103. The numerical values of the calculated conversion coefficients will be released to the public via internet as the electric database DDCC. ${ }^{8)}$ It should be mentioned that these data are different from those compiled in the forthcoming ICRP publication to be released as the revision of ICRP Publication $74,{ }^{14)}$ since the dose conversion coefficients calculated employing not only PHITS but also other simulation codes will be used in the evaluation of their reference values.

Cosmic-ray pion and muon dose rates were estimated using the calculated dose conversion coefficients for various irradiation geometries, including those closely representing the geometrical situations of the cosmic-ray muon exposure. It was found from the calculation that the dose conversion coefficients for the isotropic irradiation geometry are suitable to be used in the dose estimations for cosmic-ray pions and muons. This conclusion is the same as that derived from our previous study with respect to the neutron and proton dosimetry for aircrew. ${ }^{13)}$ The impact of the introduction of ICRP103 on the pion and muon dosimetries are negligibly small, since the $w_{R}$ values assigned to those particles are maintained in the issue.

\section{References}

1) ICRP, The 2007 Recommendations of the International Commission on Radiological Protection, ICRP Publication 103 (2007).

2) ICRP, Adult reference computational phantoms, ICRP Publication 110 (2009).

3) X. G. Xu, K. F. Eckerman, (Ed.) Handbook of Anatomical Models for Radiation Dosimetry, Boca Raton: Taylor \& Francis (2009).

4) T. Sato, A. Endo, M. Zankl et al., "Fluence-to-dose conversion coefficients for neutrons and protons calculated using the PHITS code and ICRP/ICRU adult reference computational phantoms,” Phys. Med. Biol., 54, 1997-2014 (2009).

5) T. Sato, A. Endo, K. Niita, "Fluence-to-dose conversion coefficients for heavy ions calculated using the PHITS code and ICRP/ICRU adult reference computational phantoms," Phys. Med. Biol., 55, 2235-2246 (2010).

6) K. Niita, T. Sato, H. Iwase et al., "Particle and heavy ion transport code system; PHITS,” Radiat. Meas., 41, 1080-1090 (2006).

7) K. Niita, N. Matsuda, Y. Iwamoto et al., PHITS: Particle and Heavy Ion Transport code System, Version 2.23, JAEA-Data/Code 2010-022, Japan Atomic Energy Agency (JAEA) (2010).

8) DDCC: Databases of Dose Conversion Coefficient, http://phits.jaea.go.jp/ddcc/

9) A. Ferrari, M. Pelliccioni, M. Pillon, "Fluence to Effective 
Dose Conversion Coefficients for Muons,” Radiat. Prot. Dosim., 74, 227-233 (1997).

10) A. Ferrari, M. Pelliccioni, M. Pillon, "Fluence to Effective Dose Conversion Coefficients for Negatively and Positively Charged Pions," Radiat. Prot. Dosim., 80, 361-370 (1998).

11) ICRP, 1990 Recommendations of the International Commission on Radiological Protection, ICRP Publication 60 (1991).

12) T. Sato, H. Yasuda, K. Niita et al., "Development of PARMA: PHITS-based analytical radiation model in the atmosphere,"
Radiat. Res., 170, 244-259 (2008);

http://phits.jaea.go.jp/expacs/

13) T. Sato, A. Endo, M. Zankl et al., "Fluence-to-dose conversion coefficients for aircrew dosimetry based on the new ICRP recommendations," Prog. Nucl. Sci. Technol., 1, 134-137 (2011).

14) ICRP, Conversion coefficients for use in radiological protection against external radiation, ICRP Publication 74 (1996). 